\title{
Acute hematogenous osteomyelitis due to Salmonella in a pediatric patient: a case report
}

\begin{abstract}
Acute hematogenous osteomyelitis is an inflammatory process in the bone tissue. It's usually of bacterial etiology, caused by a contiguous infection focus. In pediatric patients, the main isolated pathogen is Staphylococcus aureus. Salmonella spp. as a causative agent of osteomyelitis is infrequent; it can be found in less than $1 \%$ of the casesand it is generally associated with risk factors.

We analyze the case of a 4 years old male patient known to be healthy, who enters the emergency service with a 2 weeks of increasing fever and difficulty when walking. Upon physical examination, he presented acute pain, edema and a local increased temperature in the right ankle. He was hospitalized for suspicion of septic arthritis and he was treated empirically with clindamycin. Positive cultures were reported for Salmonella spp, isolated from blood and a tibia's secretion sample. The betalactambased approach, specifically third-generation cephalosporins, is recommended in international treatment guidelines, so the success of the use of ampicillin was expected. Thus, identifying the etiological agent of the infectious process is determinant in the success of antibiotic therapy, as well as the joint approach of the medical personnel and the laboratory service.
\end{abstract}

Keywords: acute hematogenous osteomyelitis, Salmonella spp, antibiotic therapy

\author{
Volume 3 Issue 6 - 2018
}

\author{
Lizbeth Alfaro-Arroyo,' Gabriela Cruz- \\ Chavarría, ${ }^{2}$ Lorely Blanco-Chaves, ${ }^{3}$ Andrea \\ Rodriguez-Díaz, ${ }^{3}$ Laura Blanco-Quirós ${ }^{4}$ \\ 'Head of Inmunology Section, Microbiologist and Clinical \\ Chemistry, San Carlos Hospital, Costa Rica \\ ${ }^{2}$ Third shift Chief, Microbiologist and Clinical Chemistry, Student \\ of the Specialty Postgraduate in Medical Bacteriology, University \\ of Costa Rica, Costa Rica \\ ${ }^{3}$ Microbiologist and Clinical Chemistry, San Carlos Hospital, \\ Costa Rica \\ ${ }^{4} \mathrm{Head}$ of Bacteriology Section, Microbiologist and Clinical \\ Chemistry, Specialist in Medical Bacteriology at the University \\ of Costa Rica, Costa Rica
}

\begin{abstract}
Correspondence: Ana Gabriela Chavarria,Third shift Chief, San Carlos Hospital, Microbiologist and Clinical Chemistry, Student of the Specialty Postgraduate in Medical Bacteriology at the University of Costa Rica, Alajuela, Costa Rica, Email ana.grcuz73@gmail.com
\end{abstract}

Received: September 26, 2018 | Published: November 0I, 2018

\section{Introduction}

Acute osteomyelitis is defined as an inflammatory process in the bone tissue, usually of bacterial etiology. It is classified according to its evolution and pathogenesis in: hematogenous acute osteomyelitis, caused by a contiguous infection focus and secondary to vascular insufficiency. ${ }^{1}$ In pediatric patients, it is most common disseminated by blood, where Staphylococcus aureus is the main isolated pathogen. ${ }^{2}$ Different clinical conditions have been described in the infection by Salmonella spp; gastroenteritis, enteric fever, septicemia, localized infections and asymptomatic colonization. The most frequent form of salmonellosis is gastroenteritis with a spontaneous resolution generally. ${ }^{3}$

Salmonella spp. as a causative agent of osteomyelitis is infrequent, it can be found in less than $1 \%$ of cases and usually when it occurs is associated with risk factors such as anemia, immunosuppression or connective tissue diseases. ${ }^{4}$

The purpose of the analysis of this clinical case is to study the approach in the treatment of acute hematogenous osteomyelitis caused by Salmonellaspp and the protocols for changing antibiotic therapy.

\section{Case report}

Male patient, 4 years old, previously healthy, presented fever of $39^{\circ} \mathrm{C}$ for two weeks and pharyngotonsillitis treated with antibiotics. Patient was admitted to the emergency service on July $18^{\text {th }}, 2018$. The physical examination foundhimconscious, irritable, afebrile, eupneic, hydrated, oropharynx with mild hyperemia. He was having dificulties towalk since 5 days ago, also acute pain, edema and local increased temperature in right ankle. Blood tests were performed and showed red blood cell (RBC): hemoglobin $10.9 \mathrm{~g} / \mathrm{dL}$ and white blood cell (WBC): 23130cells/uL; neutrophils: 51\%, lymphocytes: $39 \%$ and monocytes:
9.4\%, the C-reactive protein (CRP): $9.72 \mathrm{mg} / \mathrm{dL}$ and erythrocyte sedimentation rate (ESR): $15 \mathrm{~mm} / \mathrm{h}$ and blood cultures were taken at admission. The patient was hospitalized in the pediatric service for suspected arthritis. The results of the rheumatological markers were negative so the diagnosis was oriented to a septic arthritis and was treated empirically with Clindamycin.

On July $19^{\text {th }}$, the positive blood culture was reported by Gramnegative bacilli and that same day the patient was admitted to the operating room with a diagnosis of acute hematogenous osteomyelitis, performing the drainage of the distal tibia and taking a sample of secretion for culture.

On July $22^{\text {nd }}$, the general condition of the patient was evaluated, establishing a therapeutic failure due to the presence of febrile peaks and the persistence of signs of inflammation and edema around the surgical wound. Positive blood cultures were reported for Salmonella spp isolated from blood and from the tibia's secretion sample, so in coordination with the laboratory, it was decided to change the therapy for ampicillin. Nevertheless, the patient presented diarrhea and positive toxin of Clostridium difficile was detected; hence, metronidazole was established as complementary therapy.

The patient was kept in the pediatric service with an evident positive evolution. On August $6^{\text {th }}$, he was released with trimethoprim/ sulfamethoxazole oral treatment for a month and a valuation appointment by the orthopedic service.

\section{Discussion}

Infectious arthritis can be developed in one or more joints and it can be caused by bacteria, viruses, fungi or parasites. Differential diagnosis must also be made with the sterile arthritis produced by the accumulation of uric acid crystals or immune complexes. 
Clinically acute bacterial arthritis should be differentiated from viral arthritis, produced mainly by Parvovirus B19 or Chikungunya virus; however, these infections are usually polyarticular and present in outbreaks. ${ }^{4}$ In the clinical case studied, markers of sepsis and the result of positive blood cultures within a few hours of observation of the patient oriented the diagnosis to an acute bacterial arthritis disseminated by blood. The initial antibiotic treatment is generally empirical, selecting the antibiotic depending on the patient's age and base pathology. In thiscase, clindamycin was administered as initial therapy since this drug is active against gram-positive and anaerobic bacteria, which represents of $75 \%$ of the most frequent pathogens. ${ }^{4}$ When the result of the cultures is available, antibiotic therapy is modified according to the sensitivity of the isolated microorganism. ${ }^{5}$

The finding of Salmonella spp. in the blood cultures and the biopsy of the tibia responds to a possible extraintestinal complication of an enteric fever, which probably occurs in the two weeks prior to the patient's admission. This isolation is rare and it's associated with risk factors; yet, cases have also been described in healthy patients.

The betalactam-based approach, specifically third-generation cephalosporins, is highly recommended in international treatment guidelines, ${ }^{5}$ so the success of the use of Ampicillin was fully expected.

\section{Conclusion}

By delving into the events described above, we conclude that, although there are guidelines for the choice of empirical treatment of acute hematogenous osteomyelitis, identifying the etiological agent of the infectious process is a determining factor in the success of antibiotic therapy. Therefore, a joint approach of the medical personnel and the laboratory in these cases is highly recommendable.

\section{Acknowledgement}

None.

\section{Conflict of interest}

The Authors declare no conflict of interests.

\section{References}

1. Gómez OSA. Sosa VCD. An updated view on risk factors and complications of pediatric osteomyelitis. Cuban Journal of Pediatrics. 2016;88(4):463-482.

2. Bueno BM, Ruiz JM, Ramos AJT, et al. Acute osteomyelitis: epidemiology, clinical manifestations, diagnosis and treatment. $A n$ Pediatr. 2013;78(6):367-373.

3. Murray PR. Medical microbiology. 6th ed. Editorial Elsevier Mosby. 2009:309.

4. Mandell D. Bennett's - Infectious diseases. 8th ed. Principles and practice. 2016:1359-1360.

5. Hernández SMT, Zarzoso FMLS. Navarro GMM, et al. Osteomyelitis and septic arthritis. spanish association of pediatrics. diagnostic-therapeutic protocols. Pediatric Infectious Diseases Section. 2011;205-220. 